\title{
RhoB modifies estrogen responses in breast cancer cells by influencing expression of the estrogen receptor
}

\author{
Claire Médale-Giamarchi ${ }^{1,2}$, Isabelle Lajoie-Mazenc ${ }^{1,2}$, Emilie Malissein ${ }^{1}$, Elise Meunier ${ }^{1}$, Bettina Couderc ${ }^{2,3}$, \\ Yann Bergé ${ }^{4}$, Thomas Filleron ${ }^{4}$, Laura Keller ${ }^{1}$, Claudine Marty ${ }^{4}$, Magali Lacroix-Triki ${ }^{4}$, Florence Dalenc ${ }^{1}$, \\ Sophie F Doisneau-Sixou ${ }^{1,2,5^{*}}$ and Gilles Favre ${ }^{1,2^{*}}$
}

\begin{abstract}
Introduction: RhoB has been reported to exert positive and negative effects on cancer pathophysiology but an understanding of its role in breast cancer remains incomplete. Analysis of data from the Oncomine database showed a positive correlation between $R h o B$ expression and positivity for both estrogen receptor alpha (ER $\alpha$ ) and progesterone receptor (PR).

Methods: This finding was validated by our analysis of a tissue microarray constructed from a cohort of 113 patients and then investigated in human cell models.

Results: We found that RhoB expression in tissue was strongly correlated with ERo and PR expression and inversely correlated with tumor grade, tumor size and count of mitosis. In human breast cancer cell lines, RhoB attenuation was associated with reduced expression of both $E R \alpha$ and PR, whereas elevation of RhoB was found to be associated with ER $\alpha$ overexpression. Mechanistic investigations suggested that RhoB modulates ER $\alpha$ expression, controlling both its protein and mRNA levels, and that RhoB modulates PR expression by accentuating the recruitment of $E R \alpha$ and other major co-regulators to the promoter of $P R$ gene. A major consequence of RhoB modulation was that RhoB differentially regulated the proliferation of breast cancer cell lines. Interestingly, we documented crosstalk between RhoB and $E R \alpha$, with estrogen treatment leading to RhoB activation.

Conclusion: Taken together, our findings offer evidence that in human breast cancer RhoB acts as a positive function to promote expression of ER $\alpha$ and PR in a manner correlated with cell proliferation.
\end{abstract}

\section{Introduction}

Hormone therapy is recommended in breast cancers that express estrogen receptor alpha $(\mathrm{ER} \alpha)$ and/or progesterone receptor (PR). This therapy is largely effective but there are nevertheless many cases of systemic resistance. A number of studies have addressed the question of the mechanisms of resistance to hormone therapy [1,2]. ER $\alpha$ transcriptional effects are not only determined by ligands as estradiol $\left(E_{2}\right)$, but also by crosstalk between ER $\alpha$ and growth factor signaling [3]. The hierarchy among these

\footnotetext{
* Correspondence: doisneau-sixou.sophie@claudiusregaud.fr; favre. gilles@claudiusregaud.fr

'INSERM U563 and UMR1037, Institut Claudius Regaud, 20-24 rue du pont St Pierre, Toulouse cedex 31052, France

Full list of author information is available at the end of the article
}

associations is not known and various growth factor receptors are likely to be required [3].

Prenylated proteins such as Rho GTPases are key elements in growth factor signal transduction pathways [4]. A variety of growth factors present in the tumor microenvironment activate Rho proteins [5], especially RhoB [6-8]. As a Rho protein, RhoB cycles between GTP and GDP bound states, forming interactions with a variety of effectors that modulate activity and influence important processes in cancer [9]. RhoB, in contrast to its relatives RhoA and RhoC, has been shown to function as a tumor suppressor gene on the basis of investigations of genetically RhoB-deficient strains [10] and in human cancer cells [11-13]. RhoB is an immediate early response gene that is induced by a variety of stimuli, including growth

\section{Ciomed Central}


factors [6,14-21]. Although no mutation of Rho GTPases have been detected in human tumors, a correlation has been demonstrated between Rho protein overexpression and poor clinical outcome in breast cancers [22]. RhoB overexpression has been correlated to disease progression [23], although this is a controversial issue [24], and overexpression of guanine exchange factors (GEF) for Rho GTPases have been correlated to prognosis in breast cancers [25]. Indeed, breast tumor progression is accompanied by a decrease in expression of the pro-oncogenic RhoGEF Tiam1 [26]. Moreover, expression of Rho-GDI $\alpha$, a negative regulator of Rho proteins, is reported to correlate with the outcome of patients with breast cancer treated by adjuvant chemotherapy [27]. From a molecular point of view, scaffold proteins involved in Rho functions, such as Rho-GDI or Dblx, have been observed as part of ER $\alpha$-containing complexes [28,29] with direct interaction between ER $\alpha$ and Rho-GDI [30].

Besides the suggested specific involvement of RhoB in ER signaling, there have been no detailed investigations in breast cancer cells, including the assessment of any correlation with the expression of hormone receptors in tumors. A major goal of the present study was therefore to determine the involvement of RhoB in hormonedependent breast cancers and to investigate hypothesized crosstalks between RhoB and ER $\alpha$ signaling.

\section{Materials and methods}

\section{Immunohistochemical analysis of tissue microarrays}

A tissue microarray was constructed from the 113 patients described in Table S1 in Additional file 1. As detailed in Additional file 2, the clinical trial was conducted about 30 years ago with no consent required at that time. Cores $(600 \mu \mathrm{m}$ diameter $)$ of histologically confirmed invasive breast carcinomas were extracted from the original paraffin blocks and re-embedded into a gridded recipient paraffin block using a tissue arrayer (Alphelys; Beecher Inc., Plaisir, France). For each case, three tumor cores and one normal breast core were taken from the original block.

Tissue microarray immunostaining was performed on a Techmate Horizon ${ }^{\text {TM }}$ slide processor (Dako, Trappes, France \{\}$)$. Sections were incubated with antibodies to ER $\alpha$ (NCL-L-ER-6F11; Novocastra, Nanterre, France), PR (PgR636 clone; Dako) and RhoB (sc-180; Santa Cruz Biotechnology, Heidelberg, Germany). The ER $\alpha$ and PR status was classified by immunohistochemistry expression as positive ( $\geq 10 \%$ immunoreactive cells) or negative $(<10 \%$ immunoreactive cells), according to the standards applied in France, as recommended by the Groupe d'Evaluation des Facteurs Pronostiques par Immunohistochimie dans les Cancers du Sein. RhoB immunostaining was analyzed by evaluation of the percentage of tumor-stained cells and staining intensity, allowing assessment of an ImmunoReactive Score:

$$
\text { IRS }=\% \text { score } \times \text { intensity score }
$$

Correlation of RhoB expression with clinical variables was assessed using Mann-Whitney and Spearman's rank tests. Univariate survival analysis was performed for disease-free survival by applying the log-rank test to RhoB expression levels stratified by median value. The KaplanMeier method was used to link the disease-free survival according to RhoB expression in the tumors.

\section{Cell culture and reagents}

The human breast adenocarcinoma cell lines MCF-7, ZR75, T47D, SK-BR-3 and MDA-MB-231 were obtained from the ATCC (Molsheim, France). Cells were grown routinely in DMEM (Lonza Levallois-Perret, France), supplemented with $5 \%$ fetal bovine serum (FBS; Pan-Biotech, Aidenbach, Germany). Hormonoresistant LCC2 cells (R Clarke, Karmanos Cancer Center, provided the parent MCF-7) were grown in F-12 (Lonza) phenol red-free medium, containing 5\% dextran-coated charcoal (DCC)-treated FBS. Cryopreservation of cell cultures ranged from passage 1 to 10 . Cells were used for experiments during up to 20 passages. Mouse embryonic fibroblasts (MEFs) were grown in DMEM with $10 \%$ FBS.

Where indicated, cells were deprived of $\mathrm{E}_{2}$ by growing them in phenol red-free medium containing 5\% DCCtreated FBS. Cells were treated with $50 \mathrm{nM} \mathrm{E}_{2}$ or $50 \mathrm{ng} /$ $\mathrm{ml}$ epidermal growth factor or $2 \mathrm{mM}$ ICI-182, 780 (Sigma-Aldrich Chimie, Lyon, France).

\section{siRNA transfection}

Transient transfection of $40 \mathrm{nM}$ siRNA was performed using Oligofectamine ${ }^{\circledR}$ (Life Technologies - Invitrogen, Saint Aubin, France) according to the manufacturer's instructions. The siRNA sequences of the oligonucleotide duplexes (Eurogentec, Angers, France) were siB1 (5'-CC GUCUUCGAGAACUAUGU-dTdT-3') and siB2 (5'-UGA UAUCCCUUGUCUGUAA-dTdT-3'), siER (5'-GGGAGA AUGUUGAAACACA-dTdT-3') and the nonspecific sequence siControl (5'-GACGUGGGACUGAAGGGGU-d TdT-3').

\section{Adenoviral constructs expressing RhoB and transduction protocol}

Replication-defective $(\Delta \mathrm{E} 1, \mathrm{E} 3)$ adenoviral vectors expressing RhoB under the transcriptional control of the CMV promoter were constructed with the Adeasy System (Qbiogen, Illkirch, France) as described previously [31]. Cells were transduced with adenoviral vectors (control empty vector or expressing RhoB) at a multiplicity of infection of 300:1. 


\section{Western blot analysis of human cell lines}

Cells were lysed and protein analyzed by SDS-PAGE [18]. Antibodies were used against RhoB (sc-180), ER $\alpha$ (sc-543) and, ER $\beta$ (sc-53494) from Santa Cruz Biotechnology, phospho-ER $\alpha\left(\operatorname{Ser}^{118}, 2511\right.$ and Ser $\left.{ }^{167}, 2514\right)$ from Cell Signaling (Saint Quentin Yvelines, France), $\beta$-actin (MAB1501) from Chemicon (Merck Millipore; Darmstadt, Germany), and secondary antibodies were anti-mouse (MP21120) and anti-rabbit (MP23145) horseradish peroxidase from Interchim (Montluçon, France) using a chemiluminescence detection kit (ECL; Pierce, Thermo Fisher Scientific, Courtaboeuf, France). Protein abundance was quantified by Image Quant TL analysis (GE Healthcare, Velizy-Villacoublay, France).

\section{Analysis of mouse embryonic fibroblasts generated from RhoB-deficient mice}

Heterozygous (+/-) and homozygous (-/-) mutant strains of RhoB-deficient mice [10] were kindly provided by $\mathrm{G}$ Prendergast (Lankenau Institute for Medical Research, Wynnewood, PA, USA). Claudius Regaud Institute animal ethics committee approval was obtained (\# ICR-2012-001A) for the use of the animal model and the study protocols. Mice were housed in polycarbonate cages in controlled conditions. MEFs were generated [10] before lysis [18]. Protein were extracted and analyzed as described above with antibody against murine ER $\alpha$ (sc-542) or RhoB (sc-180; Santa Cruz Biotechnology).

\section{GST pull-down assay}

The level of activated RhoB and GTP-bound RhoB protein was measured using the GST fusion protein containing the Rho binding domain of Rhotekin [8]. The amount of GTPbound RhoB and the total amount of RhoB in cell lysates were determined by western blot as described above [32].

\section{Immunocytochemistry}

For each condition, $5 \times 10^{5}$ cells were seeded onto glass slides dishes and were grown for 3 days in phenol red-free DMEM, containing 5\% DCC-treated FBS. Cells were then treated with $\mathrm{E}_{2}$ during 16 hours, washed in PBS and fixed in RCL2 [33] for 2 minutes. Staining was performed by a Techmate Horizon ${ }^{\mathrm{TM}}$ slide processor as described for Figure 1 . The primary antibody used was a monoclonal anti-ER $\alpha$ antibody (HC-20, sc-543, dilution 1:50; Santa Cruz Biotechnology) or an anti-PR antibody (PgR636 clone, dilution 1:50; Dako).

The level of ER $\alpha$ and PR staining was determined by evaluation of the percentage of tumor-stained cells and staining intensity, allowing assessment of an IRS.

\section{Quantitative reverse-transcribed PCR}

Total RNA was isolated 16 hours after stimulation by $E_{2}$, extracted using the RNeasy kit following the manufacturer's instructions (Qiagen, Courtaboeuf, France) and reverse-transcribed using the iScript ${ }^{\mathrm{TM}} \mathrm{cDNA}$ Synthesis Kit (Biorad, Marnes la Coquette, France). Quantitative PCR was performed with an iQreal-time PCR detection system (Biorad). The specific human primer pairs used were: for GAPDH, 5'-TGCACCACCAACTGCTTAGC-3' and 5'-G GCATGGACTGTGGTCATGAG-3'; for 28s, 5'TCGCTGGGTCTTGGATGTC-3' and 5'-AGCAGATTG TGACAG ACCATTCC-3'; for PR, 5'-CGCGCTCTAC CCTGC ACTC-3' and 5'-TGAATCCGGCCTCAGGT AGTT-3'; and for ER $\alpha$, 5'-CCACCAACCAGTGCACC ATT-3' and 5'-GGT CTTTTCGTATCCCACCTTTC-3' (Eurogentec).

\section{Luciferase assay}

Development of stable transfectants of MCF-7 cells (MELN cells) has been described previously [34]. These cells, kindly provided by P Balaguer (INSERM 540, France), were established by transfecting MCF-7 cells with ERE- $\beta$-globin-luc-SV-Neo plasmid and thus express luciferase in an estrogen-dependent manner.

Three days after siRNA transfection, MELN cells were seeded in DMEM-DCC-treated FBS during 3 days. They were then treated with $E_{2}$ or ethanol for 16 hours. Cells were then lysed in reporter lysis buffer (Promega, Charbonnières Les Bains, France). The luciferase activity was measured with luciferase assay reagent (Promega) according to the manufacturer's instructions. Protein concentration was measured to normalize the luciferase activity data.

\section{Chromatin immunoprecipitation assay}

Chromatin immunoprecipitation assays were performed with modifications of the procedure of Metivier and colleagues [35] as described elsewhere [36]. Briefly, $10^{6}$ cells were synchronized by 3 days of culture in DMEM 5\% DCC-treated FCS and treated during 1 hour with $\mathrm{E}_{2}$. Immunoprecipitation antibodies were ER $\alpha$ (sc-543), HDAC1 (sc-6299), and polII (sc-899) from Santa Cruz Biotechnology, and acetylated histone H3 (ab1791) and H4 (ab193) from Abcam (Paris, France). Quantitative PCR were performed on an iCycler (Biorad) using the primers 5'-GGCGACAC AGCAGTGGGGAT-3' and 5'-TCTCCTC CCTCTGCC CCTATATTC-3' (Eurogentec) to amplify the fragment of the human PR promoter flanking the +745 AP-1 site [37].

\section{Cell growth determination}

Forty-eight hours after siRNA transfection or transduction, cells were seeded in DMEM 5\% FBS with ethanol or $\mathrm{E}_{2}$ and counted daily during the next 4 days with a coulter counter (Beckman Coulter, Grenoble, France).

\section{Results}

RhoB expression correlates positively with receptor status and negatively with grade in human breast tumors Using the Oncomine microarray database, a correlation between $R h o B$ and either ER $\alpha$ or PR expression was 


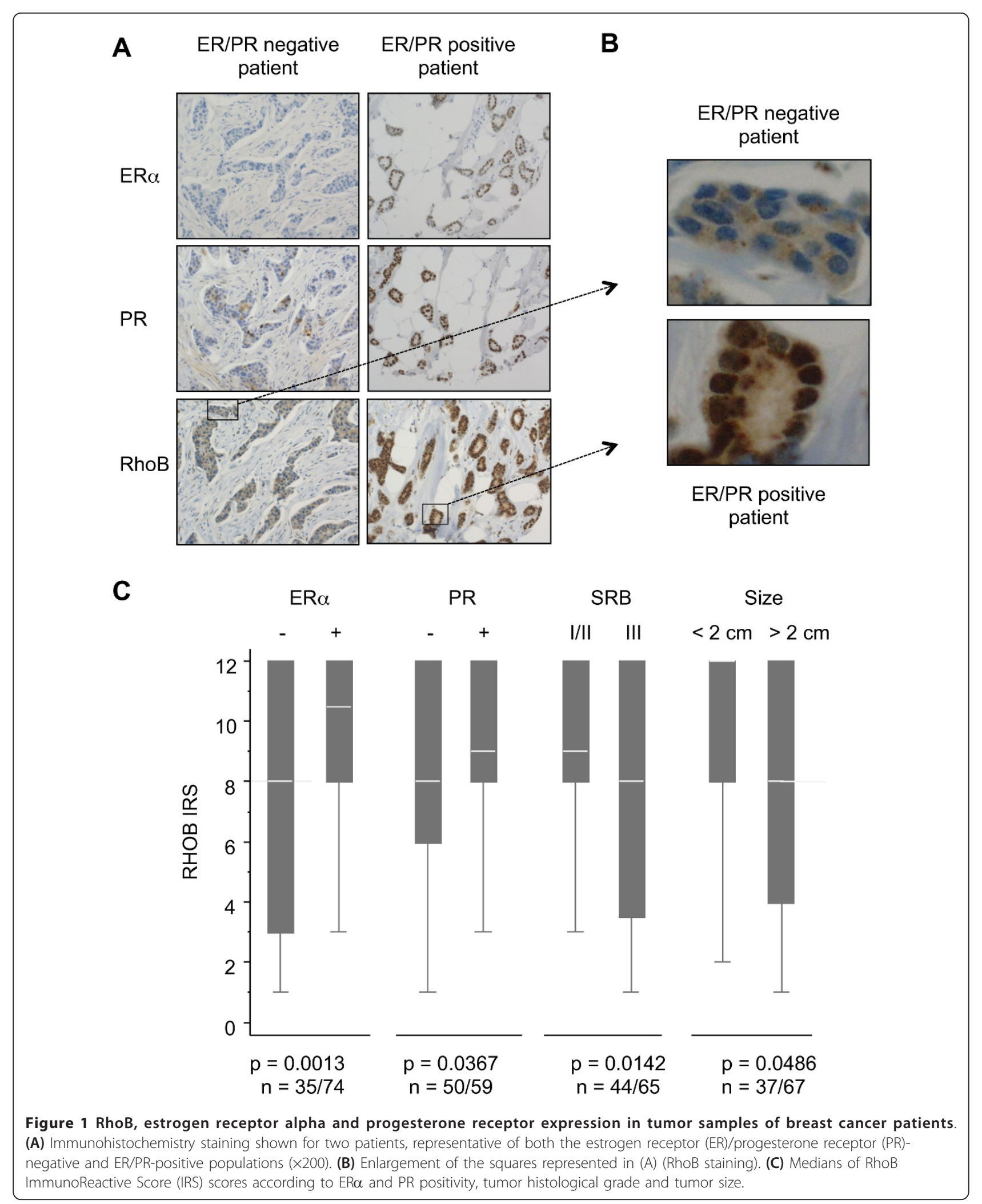


documented in datasets from 19 breast cancer studies. Moreover, four studies showed an inverse correlation between $R h o B$ expression and tumor grade. To confirm the clinical relevance of RhoB expression levels in breast cancer oncogenesis and outcome, we compared the expression levels of RhoB in breast carcinomas from a cohort of 113 patients treated or not by tamoxifen in an adjuvant setting in a randomized prospective study. Patient characteristics are described in Table S1 in Additional file 1. ER $\alpha$ and PR assessments were initially performed with biochemistry techniques at the time of diagnosis (between 1980 and 1983). They have all been performed again at the time of analysis, in parallel with RhoB assessment. After new pathological analysis, 65 tumors were classified as grade I and II, 74 were ER $\alpha$ positive, and 59 were PR-positive. Among these tumors, 23 presented a lymphovascular invasion and 39 cases presented with positive lymph nodes. After randomization, we determined that age, ER $\alpha$ and PR status, histological grade, and type and lymphovascular invasion status were similar in the two groups of patients, whether treated or not. Nonetheless, patients treated with tamoxifen $(n=62)$ had less favorable prognostic factors regarding positive lymph nodes, pathological tumor size and number of mitoses.

Figure $1 \mathrm{~A}$ shows the RhoB immunohistochemistry stainings of two representative tumors of patients displaying opposite hormone receptor status (ER $\alpha / \mathrm{PR}$-negative and $E R \alpha / P R$-positive), illustrating the direct correlation between RhoB and both ER $\alpha$ and PR expression. Enlargement of the photographs (Figure 1B) illustrates in the tumor cells of the ER $\alpha / P R$-negative patient that RhoB is present and specifically cytoplasmic, although the staining is weak. For the ER $\alpha / P R$-positive patient, in addition to cytoplasmic staining, a strong staining was also observed at nucleus level.

Statistical analysis of the results indicated that RhoB expression in tumors is strongly correlated with the percentage of ER $\alpha$ (Spearman's $\rho=0.3659, P=0.001$ ) and PR ( $\rho=0.2544, P=0.0076)$ expression, but inversely correlated with histological tumor size $(\rho=-0.2344, P=$ $0.0166)$ and number of mitoses $(\rho=-0.2009, P=0.0362)$. We divided the patients with ER $\alpha$-positive tumors into two groups with either low or high RhoB expression, and in each group we further divided the patients into groups that did or did not receive tamoxifen. This analysis argued that the level of RhoB expression was not correlated with disease-free survival of patients with ER $\alpha$-positive tumors, regardless of tamoxifen treatment or not (Kaplan-Meier curve shown in Figure S1 in Additional file 3).

The RhoB IRS (see Materials and methods) integrating both the percentage and intensity of the staining (Figure 1C) was significantly higher in ER $\alpha$-positive tumors (median 10.5 (3 to 12)) as compared with ER $\alpha$-negative tumors (median 8 (1 to 12$)$ ), in PR-positive tumors (median 9 (3 to 12)) as compared with PR-negative tumors (median 8 (1 to 12$)$ ), and in patients with tumor grade I and II (median 9 (3 to 12)) as compared with grade III (median 8 (1 to 12)). The line in the center of each box represents the median value of the distribution, and the upper and lower ends of the box are the upper and lower quartiles, respectively. The RhoB level of expression was also higher in the smaller tumor size ( $\leq 2 \mathrm{~cm}$, median 12 (2 to 12$)$ ) as compared with larger tumors ( $>2 \mathrm{~cm}$, median 8 ( 1 to 12$)$ ). The RhoB IRS score was not correlated with the presence of lymphovascular invasion $(P=0.26)$, nor with the presence of lymph node invasion $(P=0.74)$.

\section{RhoB regulates $E R \alpha$ expression in MCF-7, in vivo and in other breast cancer cell lines}

We confirmed the expectation of an effect of RhoB on $E R \alpha$ expression in MCF-7 cells in the presence of $E_{2}$, using two different siRNA sequences to target RhoB mRNA (siB1 and siB2) (Figure 2A, left panel). We observed associated decreases in ER $\alpha$ expression of $60 \%$ and $62 \%$ (siB1 and siB2, respectively). This result was confirmed using two other independent siRNA sequences targeting RhoB (data not shown).

To rule out the possibility of off-target effects of the siRNA approach, we transduced MCF-7 cells with an adenoviral vector expressing RhoB (Figure 2A, right panel). The observed 4.5-fold overexpression of RhoB increased the expression of ER $\alpha$ (186\%), further supporting the hypothesized regulatory relationship.

We extended the study of RhoB downregulation on ER $\alpha$ expression to additional cell lines (Figure S2 in Additional file 4), confirming a decrease of $E R \alpha$ expression with RhoB depletion using siB1 and siB2 in T47D and ZR75 cells (hormone-dependent cells) or in LCC2 cells (hormone-resistant cells). We confirmed the involvement of RhoB on ER $\alpha$ expression in vivo using mice that are genetically deficient in RhoB (Figure 2B). A major decrease of $E R \alpha$ expression was visualized in MEFs collected from $\mathrm{RhoB}^{+/-}$mice with an even more dramatic decrease in MEFs collected from $\mathrm{RhoB}^{-/-}$mice. These results extended the support for a hypothesized regulatory relationship between RhoB and ER $\alpha$.

We also studied the levels of ER $\alpha$ phosphorylated forms whose involvement as prognostic markers is discussed below (Figure 2C). We observed that the decrease of ER $\alpha$ expression observed when RhoB expression is downregulated is not associated with any significant specific change of P-Ser ${ }^{118}$ or P-Ser ${ }^{167} \mathrm{ER} \alpha$ level. Indeed, the ratio phosphorylated/total ER $\alpha$ is not modified after RhoB inhibition.

We then demonstrated that ER $\alpha$ expression is dramatically decreased in the presence of the pure anti-estrogen 
A
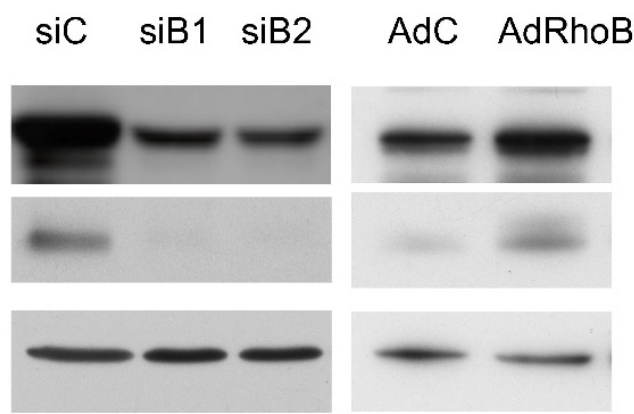

ERa

RhoB

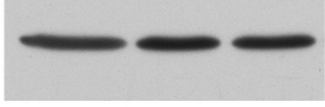

C

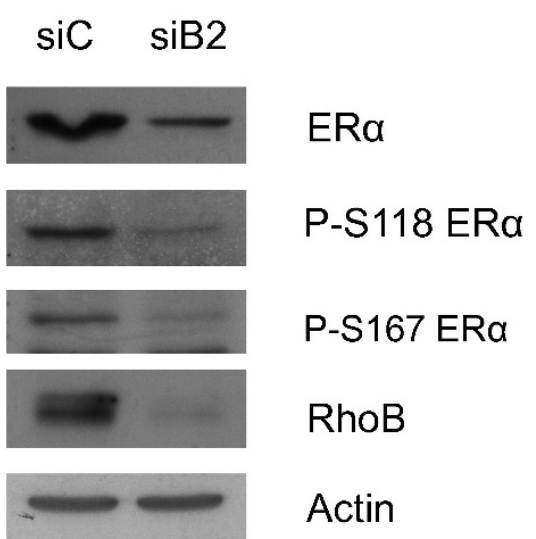

B

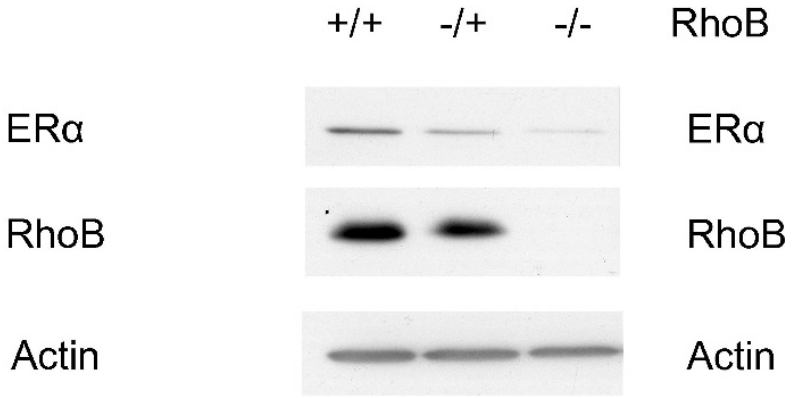

D

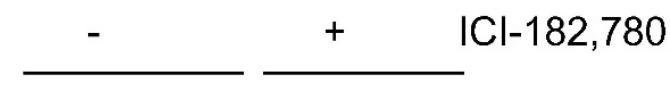

AdC AdRhoB AdC AdRhoB

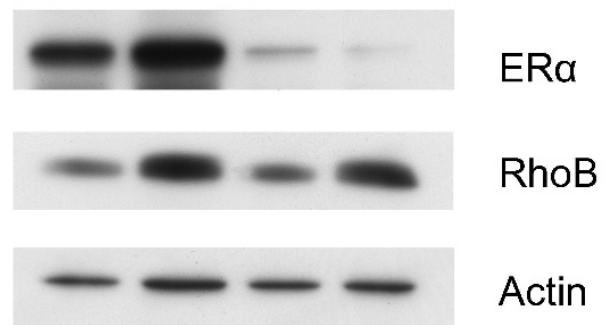

Figure 2 Regulation of estrogen receptor alpha expression by RhoB in MCF-7 cells and in vivo. (A) Cells were transfected with siControl (siC), siB1 or siB2 (left panel) or transduced with adenoviral vectors (multiplicity of infection (MOI) 300:1) (right panel) during 48 hours. (B) Mouse embryonic fibroblasts (MEFs) of RhoB-deficient mice were lysed. (C) MCF-7 cells were transfected with siC or siB2. Estrogen receptor alpha (ER $\alpha$ ) phosphorylation was analyzed 48 hours later. (D) Cells were transduced with adenoviral vectors (MOI 300:1) during 48 hours, and 3 days later were treated by $\mathrm{ICl}-182,780$ or ethanol during 16 hours. (A) to (D) Protein expression was analyzed. Representative of three independent experiments. AdC, adenoviral control empty vector.

ICI-182, 780, even when RhoB is overexpressed (Figure 2D).

\section{Estrogen induces RhoB activation}

In examining the effect of estrogen on RhoB expression and activity and given that the activation of RhoB is important for its physiological effect $[8,18,20]$, we investigated the role of estrogen stimulation on the RhoB guanine nucleotide binding status (Figure $3 \mathrm{~A}$ ) to analyze rapid effects of $E_{2}$ stimulation on RhoB activation as well as expression. We observed that RhoB is quickly activated at 30 minutes without any change of total RhoB expression, as described with epidermal growth factor stimulation $[8,20]$. At 1 hour and 2 hours of treatment, $E_{2}$ rapidly increased the total RhoB expression with a second peak of GTP-bound RhoB occurring at 2 hours associated with a parallel increase of total RhoB expression.

Together these results suggested that RhoB and estrogen signaling are integrated into a feed-forward loop that may positively modify the biological effects of estrogen treatment.

RhoB regulates $E R \alpha$, but not $E R \beta$, expression in the absence and presence of estradiol in MCF-7 cells

We analyzed further the effect of RhoB downregulation on ER $\alpha$ and ER $\beta$ expression in the absence and presence of $\mathrm{E}_{2}$ (Figure $3 \mathrm{~B}$ ). As expected, $\mathrm{E}_{2}$ treatment induced a 


\section{A}

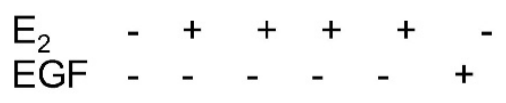

$\min \quad \begin{array}{llllll}0 & 30 & 60 & 90 & 120 & 30\end{array}$

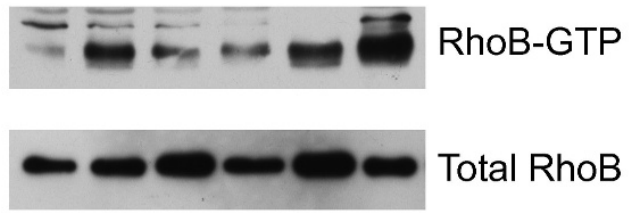

C

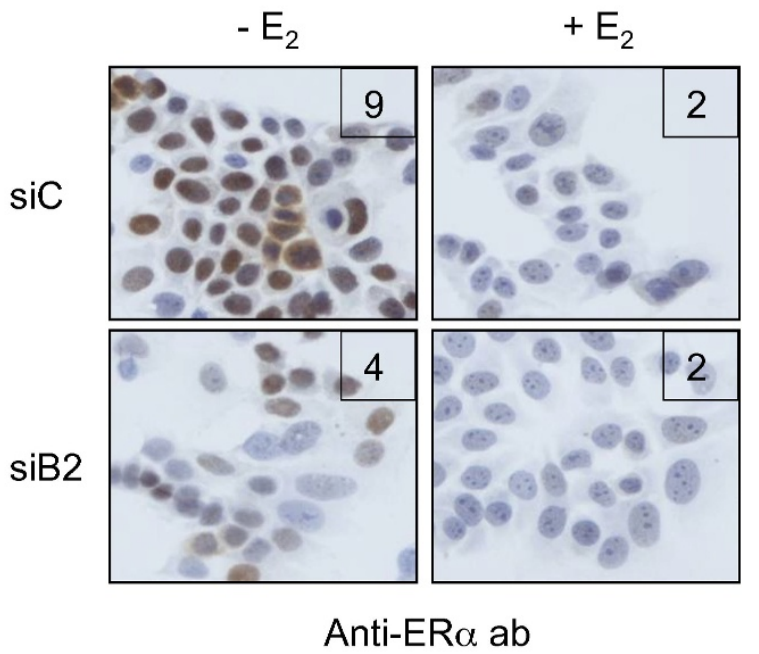

B
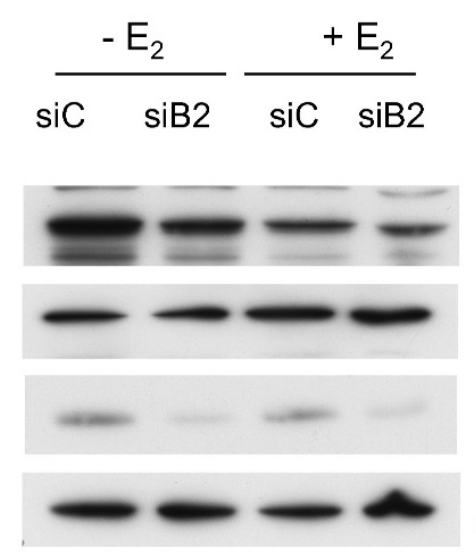

ERa

ER $\beta$

RhoB

Actin

D

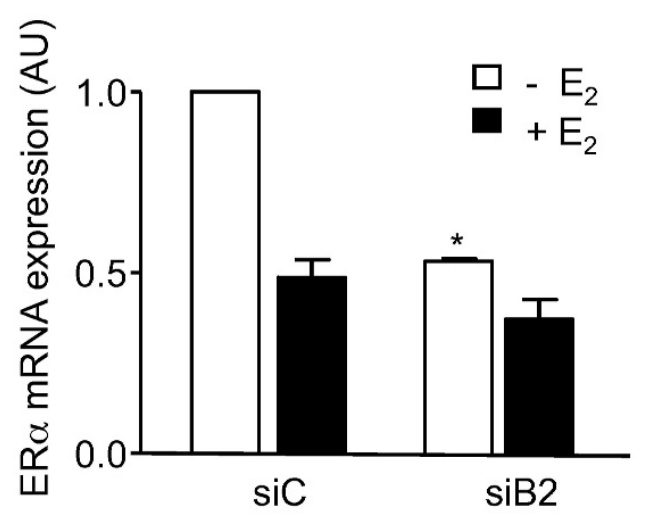

Figure 3 RhoB activation and regulation of estrogen receptor alpha expression with/without estradiol in MCF-7 cells. (A) Cells were deprived of estradiol $\left(E_{2}\right)$ for 3 days and then treated by $E_{2}$ or epidermal growth factor (EGF) during the indicated times. GTP-bound RhoB and total lysates were collected. Protein expression was then analyzed. Representative of three to four independent experiments. (B) to (D) Cells were transfected with siControl (siC) or siB2, and 3 days later were deprived of $E_{2}$ for 3 additional days and treated for 16 hours with $E_{2}$ or ethanol. (B) Protein expression was then analyzed. Representative of three independent experiments. (C) Estrogen receptor alpha (ER $\alpha$ ) expression was analyzed by immunocytochemistry. ImmunoReactive Score (IRS) shown in the upper-right corner. Representative of three independent experiments. (D) Expression of the $E R \alpha$ gene was then measured. Error bars represent the mean values \pm standard deviation from triplicate conditions, representative of two independent experiments. Differences were considered statistically significant at ${ }^{*} P<0.01$, Student's $t$ test.

major significant decrease of ER $\alpha$ expression and concomitantly of RhoB expression. In the presence of siB2, a 36 to $41 \%$ decrease of ER $\alpha$ expression was observed in the absence of $\mathrm{E}_{2}$ and 48 to $52 \%$ in the presence of $\mathrm{E}_{2}$. In contrast, ER $\beta$ expression was not clearly altered by RhoB downregulation (Figure 3B). Using immunocytochemistry, we confirmed these results suggesting that RhoB regulates $E R \alpha$ expression and observed no dramatic changes of RhoB subcellular localization within MCF-7 cells (Figure 3C). $\mathrm{ER} \alpha$ was highly concentrated in the nucleus of the untreated control with a significant staining of the related cytoplasm. In the presence of $E_{2}$, the staining intensities of both nuclei and cytoplasms were clearly decreased with no more detectable staining in the cytoplasm, confirmed by IRS assessment. For siB2-treated cells, a similar major decrease of the labeling intensity was observed in the 
cytoplasm and the nucleus, both in the presence and the absence of $E_{2}$. In the presence of $E_{2}$, the IRS score was maintained at a value of 2 because, in spite of the difference from 30 to $20 \%$ of stained cells induced by siB2 treatment, the intensity is kept very low in both cases (value of 1 ).

We then investigated whether RhoB downregulation modulates ER $\alpha$ mRNA expression (Figure 3D). We first confirmed the expected decrease of ER $\alpha$ mRNA expression in control cells treated by $\mathrm{E}_{2}$ alone (to 0.49). In the cells treated with siB2, a dramatic decrease of ER $\alpha$ mRNA was observed in the absence of $E_{2}$ (from 1 to 0.53 ) and much lighter in the presence of $E_{2}$ (from 0.49 to 0.37 ).

\section{RhoB promotes $E R \alpha$ transcriptional activation function induced by estrogen}

We hypothesized that the ability of RhoB to modulate ER $\alpha$ expression could control the major transcriptional functions of ER $\alpha$. To examine this hypothesis, we used MELN cells that express luciferase in an estrogen-dependent manner. After siB1 or siB2 transfection and $E_{2}$ deprivation, cells were treated or not with $\mathrm{E}_{2}$ and luciferase activity was quantified (Figure 4A). In the absence of $\mathrm{E}_{2}$ (upper-right corner), the transfection of cells by both siB1 and siB2 induced a significant decrease of the luciferase activity (0.5-fold for B1 and 0.7-fold for B2). In the control cells, $\mathrm{E}_{2}$ treatment led to a ninefold induction of the luciferase activity. In the presence of $\mathrm{E}_{2}$, luciferase expression was significantly decreased for cells treated by the siB1 and siB2 sequences (respectively 3.12 and 4.07 compared with 9 AU). Nonetheless, $E_{2}$ induction was still observed for all cells transfected by either siB1 or siB2. We extended the analysis of RhoB inhibition on two known estrogenregulated genes, $E R \alpha$ itself described above (Figure 3D) and $P R$, using quantitative RT-PCR (Figure 4B).

Regarding $P R$, a major target gene of $E R \alpha, P R$ mRNA expression was induced in the presence of $E_{2}$ (7.7-fold) in the siRNA control cells (Figure 4B). In cells treated with siB1, there was a significant decrease of PR mRNA levels as compared with their respective control, both without and with $\mathrm{E}_{2}$ (rates 0.5 and 0.6 , respectively). Consequently, $\mathrm{E}_{2}$ induction was maintained for cells transfected by both siRNA control and siB1 (respectively 7.7-fold and ninefold).

Using immunocytochemistry, we confirmed that RhoB regulates ER $\alpha$ (Figure 3C) plus PR (Figure 4C) expression, with no dramatic changes of their subcellular localization within MCF-7 cells. PR is mainly detected in the nuclei of the untreated control. Besides, the presence of siB2 again significantly decreases IRS scores both in the absence and presence of $\mathrm{E}_{2}$.

Cells treated with siB2 exhibit low levels of ER $\alpha$ protein and mRNA in both the absence and presence of $E_{2}$ (Figure 3B, C, D). Nonetheless, the effects of RhoB on PR expression may be supported in part by other mechanisms. We used chromatin immunoprecipitation analysis to study the effect of RhoB inhibition on the recruitment of ER $\alpha$, ER transcriptional co-factors and RNA polymerase II onto $P R$ gene promoter 1 hour after $\mathrm{E}_{2}$ stimulation (Figure 4D). Following siB1 transfection, the recruitment of RNA polymerase II to the $P R$ gene promoter dramatically decreased (rate 0.3 compared with siControl). Recruitment of acetylated $\mathrm{H} 3$ and $\mathrm{H} 4$ histones were also clearly decreased by RhoB downregulation (rates 0.36 and 0.33 , respectively). Moreover, RhoB downregulation induced a significant decrease in the recruitment of ER to the $P R$ gene promoter (rate 0.52 ) paralleled by an increased in recruitment of the ER $\alpha$ corepressor HDAC1 (rate 1.67). Together, these results indicate that RhoB may support to some extent ER $\alpha$ transcriptional activation function by interfering with its cofactor recruitment, besides the direct effect on ER $\alpha$ expression itself.

\section{RhoB induces proliferation in ER-positive but not in ER-negative breast cancer cell lines}

The effect of RhoB on cell proliferation was evaluated in three cell lines exhibiting variable levels of expression of

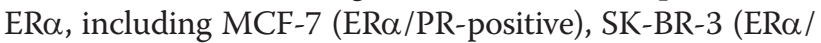
PR-negative and p185 $5^{\text {erbB2 }}$ overexpressed) and MDA-MB231 (ER $\alpha / P R-n e g a t i v e ~ a n d ~ p 185^{\text {erbB2 }}$-negative). As shown in Figure $5 \mathrm{~A}, \mathrm{~B}$, RhoB positively regulated the proliferation of MCF-7 cells both in the absence or presence of $E_{2}$. siRNA-mediated inhibition of RhoB expression produced a 30 to $35 \%$ decrease in MCF-7 cell proliferation as soon as 1 day after transfection, with a 40 to $46 \%$ decrease by day 4 (Figure 5A). Conversely, a significant increase in cell proliferation was observed in MCF-7 cells transduced with an adenoviral vector expressing RhoB (Figure 5B), with an increase of 15 to $28 \%$ in relative cell proliferation at day 1 that reached 22 to $49 \%$ by day 4 . In contrast to these observations, under similar conditions for infection of SKBR-3 or MDA-MB-231 cells, the adenoviral RhoB vector either slightly decreased or had no significant biological effect on cell proliferation (Figure 5C, D). The effect of RhoB downregulation was also analyzed in LCC2 cells, an $\mathrm{E}_{2}$-independent, tamoxifen-resistant subline of the MCF-7 cells. As for the MCF-7 cells, a significant decrease of proliferation was observed at day 4, in parallel to ER $\alpha$ and RhoB downregulation (see Figures S2 and S3 in Additional files 4 and 5).

In conclusion, we documented that RhoB had stimulatory effects on proliferation via $\mathrm{ER} \alpha$ signaling that paralleled its effects on hormone receptor expression.

\section{Discussion}

Although the tumor suppressor function of RhoB has been documented in many human cancers [11,12], RhoB overexpression was suggested to be associated with 


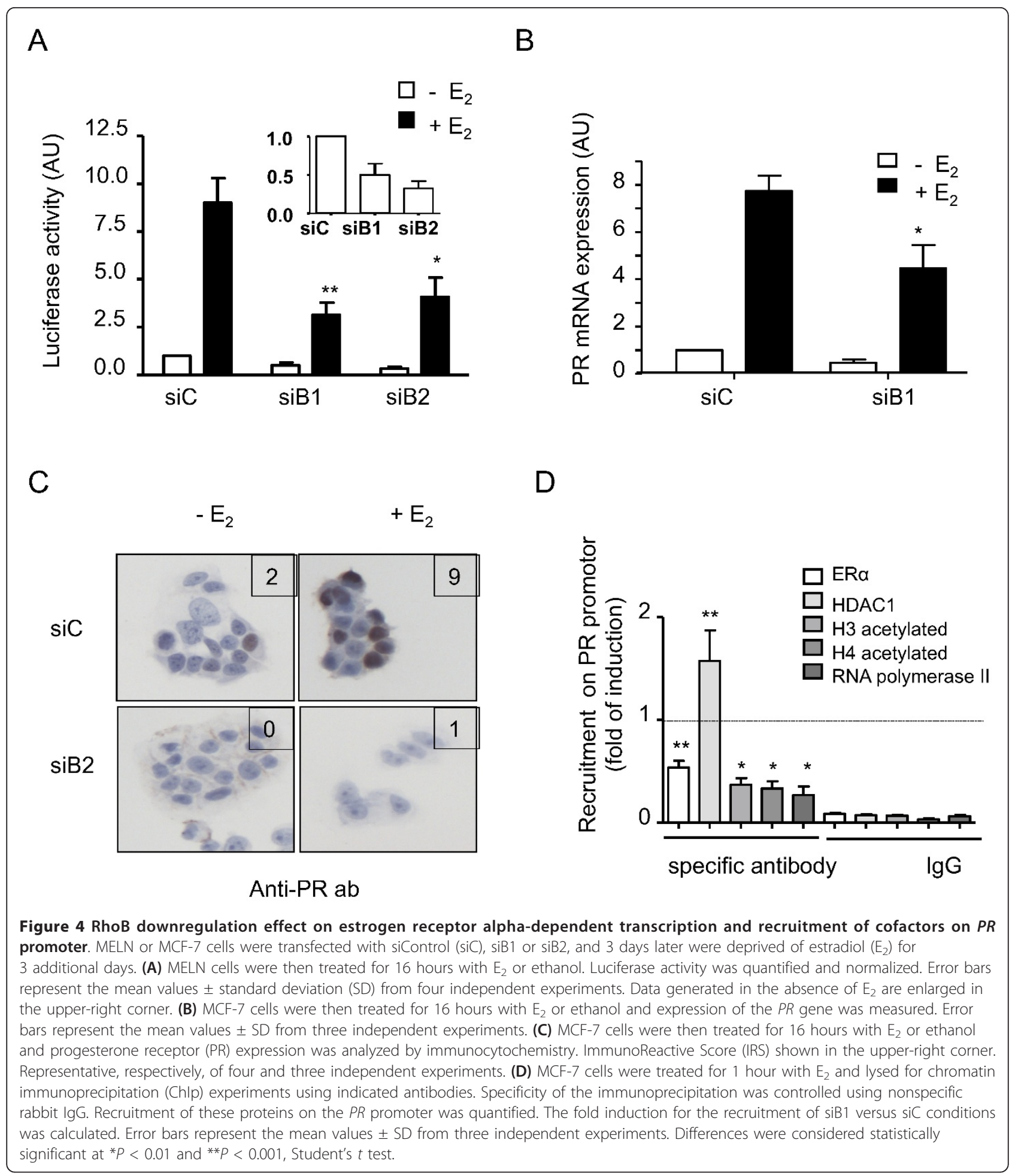

tumor progression in breast cancers. Using cellular and human breast tumor analytical approaches, we have shown a positive crosstalk between RhoB and ER $\alpha$ expression and the critical role of RhoB in regulation of the proliferation of ER $\alpha$-expressing breast cancer cells.
Our data bring together three lines of evidence to support the crosstalk between ER $\alpha$ and RhoB. First, in human breast cancer tissues we clearly showed a strong correlation between RhoB expression and the expression of ER $\alpha$ and PR. Moreover RhoB expression was associated with a 


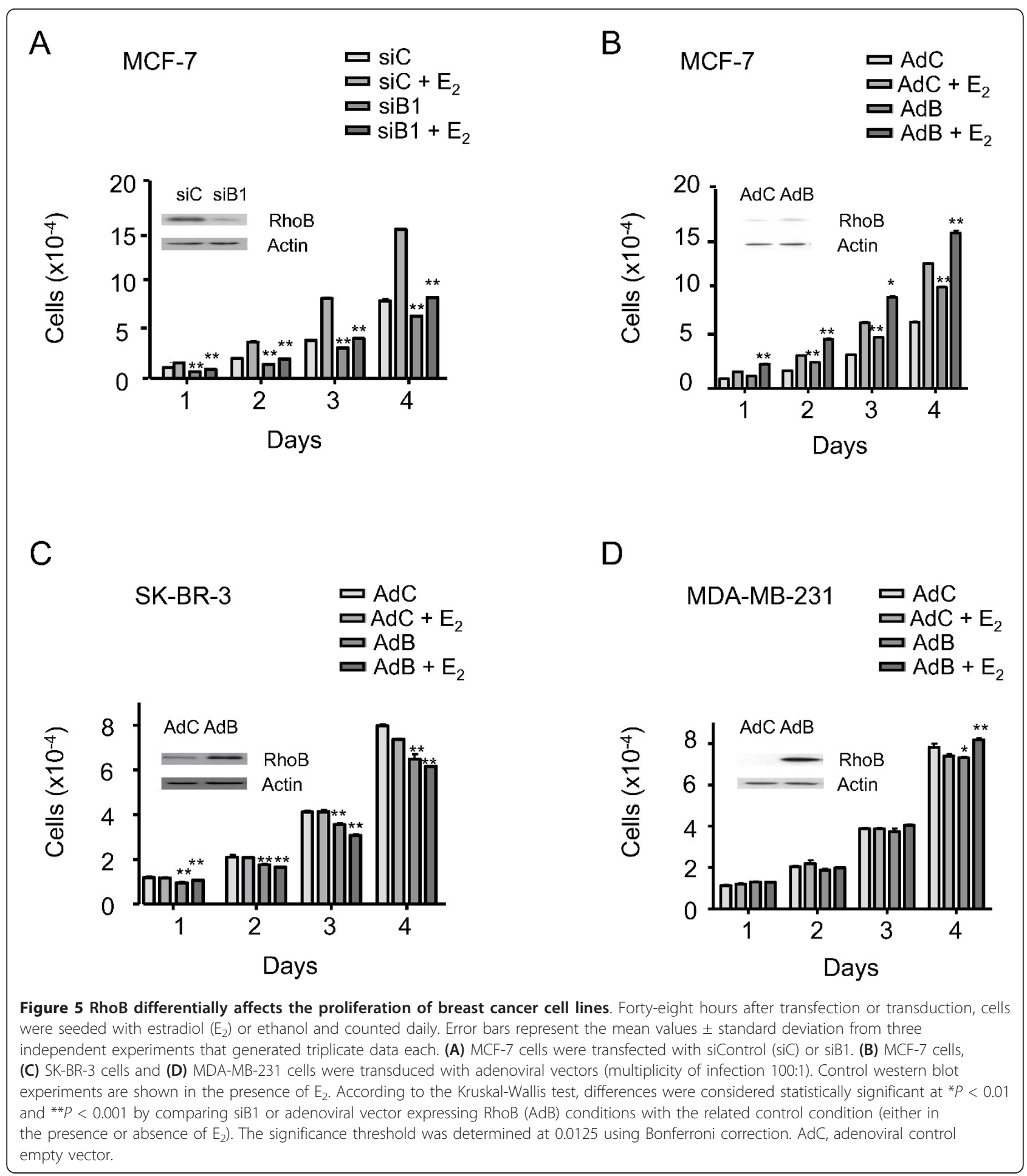

low tumor grade and size, suggesting that RhoB expression is correlated with good prognosis markers. The second strand of evidence came from cellular results showing that the level of RhoB controls the expression of ER $\alpha$ in ER $\alpha$ positive breast cancer cell in the presence or absence of $E_{2}$. The positive modulation of ER $\alpha$ expression by RhoB was evidenced both at the protein and mRNA levels. We showed that the ER $\alpha$ mRNA level is controlled by RhoB, suggesting that transcriptional regulation could play a key role in this regulation. The phosphorylation of $\mathrm{Ser}^{167}$ of $E R \alpha$ in tumors has been related to longer overall survival whereas the phosphorylation of Ser ${ }^{118}$ could be a good 
prognostic marker $[38,39]$. We found a dramatically decreased level of ER $\alpha$ phosphorylation at both serine sites that can be attributed to the major decrease in total ER $\alpha$ expression (RhoB downregulation induced no significant change in the phosphorylated/total ER $\alpha$ ratio). Third, we clearly demonstrated in vivo the RhoB control of ER $\alpha$ expression in MEF cells derived from a $\mathrm{RhoB}^{-/-}$mouse model. This regulation is of interest in the context of the epithelial-stromal interactions, particularly given that breast adipose fibroblasts determine the expression of aromatase [40]. Further, we demonstrated that ER $\alpha$ controls the activation and expression of RhoB. Notably, $E_{2}$ treatment induces an increase of active GTP-RhoB within $30 \mathrm{~min}$ utes, without changing RhoB expression at that time.

$E R \alpha$ upregulates or downregulates the transcription of hundreds of genes [41] and $P R$ is a well-known ER $\alpha$ target gene with a major physiological role in cell proliferation. Both PR mRNA and protein expression were significantly decreased as a consequence of RhoB and related ER $\alpha$ downregulations. Further findings evidenced a clear decrease of the recruitment of RNA polymerase II, acetylated $\mathrm{H} 3 / \mathrm{H} 4$ histones and $\mathrm{ER} \alpha$ onto the promoter of the $P R$ gene. In parallel, we described the recruitment of the major transcriptional co-repressor HDAC1, which is known to repress RhoB expression [42]. These results provide the demonstration of a regulatory role for RhoB in $E R \alpha$ expression and in the balance of the associated coregulators of $E R \alpha$ to control transcription of its target genes. The possibility of a direct interaction of RhoB with the ER $\alpha$-dependent transcriptional machinery should not be excluded since a direct interaction between ER $\alpha$ and RhoGDI $\alpha$ in breast cancer cells has been demonstrated [30] and we have shown here a strong nuclear localization of RhoB in ER $\alpha$-positive tumor tissues.

In contrast to other cancer models, RhoB is critical for the proliferation of ER $\alpha$-expressing breast cancer cells, suggesting its role as a positive regulator in this model. It is noteworthy that the RhoB effect is also observed on the proliferation of the ER $\alpha$-positive, tamoxifen-resistant LCC2 model cell line. Inversely, in ER $\alpha$-negative cell lines (SK-BR-3 and MDA-MB-231), RhoB has no effect on proliferation - thus reinforcing the idea that RhoB promotes cell proliferation through ER $\alpha$ expression. These results suggest that RhoB downregulation in breast cancer cells could be associated with tumor progression in parallel to ER $\alpha$ extinction, with a chronology that remains to be elucidated.

\section{Conclusion}

We have demonstrated that RhoB GTPase is a key inducer of ER $\alpha$ and a key regulator of PR expression. RhoB acts through various complex mechanisms underlying a feed-forward loop that may control estrogen effects, including cell proliferation. Our new findings shed light on the role of $\mathrm{RhoB}$ in tumorigenesis involving a dual effect conferred by cellular context with a potential prooncogenic function in hormone-dependent breast cancer cells.

\section{Additional material}

Additional file 1: Table S1 presenting clinicopathological
characteristics of 113 breast cancer patients.
Additional file 2: A word file presenting the supplementary
materials and methods, with information for the patient population
and the proliferation determination in LCC2 cells.
Additional file 3: Figure S1 showing Kaplan-Meier representation of
DFS for the patients according to RhoB expression (low or high) in
their tumors.
Additional file 4: Figure S2 showing RhoB downregulation is
associated with decreases of ER $\alpha$ expression in three other breast
cancer cell lines. T47D, ZR75 and LCC2 cells were transfected with
siControl (siC), siB1 or siB2 during 48 hours. Protein expression was then
analyzed. Representative of two to three independent experiments.
Additional file 5: Figure S3 showing RhoB downregulation is
associated with decreased proliferation in LCC2 cells. The LCC2 cells
were transfected with siControl (siC) or siB1, and the cells were seeded
48 hours after transfection and counted at day 4 . Error bars represent the
mean values \pm standard deviation from triplicate data. Representative of
two independent experiments.

\section{Abbreviations}

DCC: dextran-coated charcoal; DMEM: Dulbecco's modified Eagle's medium; $E_{2}$ : estradiol; ER: estrogen receptor; FBS: fetal bovine serum; IRS: ImmunoReactive Score; MEF: mouse embryonic fibroblast; PCR: polymerase chain reaction; PR: progesterone receptor; RT: reverse transcriptase; siRNA: small interfering RNA.

\section{Authors' contributions}

GF and SFD-S are co-last authors of this work. CM-G and IL-M carried out the cell line experiments and reviewed the manuscript, EMa carried out western blot, luciferase and GST pull-down experiments and participated in drafting the manuscript. EMe carried out chromatin immunoprecipitation, RT-PCR, western blot and proliferation experiments and participated in drafting the manuscript. BC participated in the adenovirus experiments. YB participated in immunocytochemistry experiments. TF performed all statistical analysis. LK carried out the patient data collection. CM carried out the tissue microarray. ML-T performed the tissue microarray analysis and reviewed the manuscript. FD participated in the design of the study and reviewed the manuscript. SFD-S conceived the study, coordinated the study and drafted the manuscript. GF coordinated the study and reviewed the manuscript. All authors read and approved the final manuscript.

\section{Competing interests}

The authors declare that they have no competing interests.

\section{Acknowledgements}

This work was supported by the National Institute of Cancer, Canceropole Grand Sud-Ouest, Fondation RITC (C Silvagni), INSERM, Claudius Regaud Institute, University of Toulouse (UPS) and the French Ministry of Research (MRES). Thanks are due to GC Prendergast for fruitful discussion and reviewing the manuscript.

\section{Author details}

${ }^{1}$ INSERM U563 and UMR1037, Institut Claudius Regaud, 20-24 rue du pont St Pierre, Toulouse cedex 31052, France. ${ }^{2}$ Faculté des Sciences Pharmaceutiques, Université Paul Sabatier Toulouse III, Toulouse cedex 31062, France. ${ }^{3} E A 4553$, Institut Claudius Regaud, 20-24 rue du pont St Pierre, Toulouse cedex 31052, France. ${ }^{4}$ Département de Biologie et de 
Pathologie, Institut Claudius Regaud, 20-24 rue du pont St Pierre, Toulouse cedex 31052, France. ${ }^{5}$ Tumorbiologischen Labor, Klinikum der LudwigMaximilians-Universität, Maistraße 11, München 80337, Germany.

Received: 6 August 2012 Revised: 22 November 2012

Accepted: 10 January 2013 Published: 22 January 2013

\section{References}

1. Musgrove EA, Sutherland RL: Biological determinants of endocrine resistance in breast cancer. Nat Rev Cancer 2009, 9:631-643.

2. Miller TW, Balko JM, Arteaga CL: Phosphatidylinositol 3-kinase and antiestrogen resistance in breast cancer. J Clin Oncol 2011, 29:4452-4461.

3. Fox EM, Andrade J, Shupnik MA: Novel actions of estrogen to promote proliferation: integration of cytoplasmic and nuclear pathways. Steroids 2009, 74:622-627.

4. Guilluy C, Garcia-Mata R, Burridge K: Rho protein crosstalk: another social network? Trends Cell Biol 2011, 21:718-726.

5. Jaffe $A B$, Hall A: Rho GTPases in transformation and metastasis. Adv Cancer Res 2002, 84:57-80.

6. Jahner $\mathrm{D}$, Hunter $\mathrm{T}$ : The ras-related gene rhoB is an immediate-early gene inducible by v-Fps, epidermal growth factor, and platelet-derived growth factor in rat fibroblasts. Mol Cell Biol 1991, 11:3682-3690.

7. de Cremoux P, Gauville C, Closson V, Linares G, Calvo F, Tavitian A, Olofsson B: EGF modulation of the ras-related rhoB gene expression in human breast-cancer cell lines. Int J Cancer 1994, 59:408-415.

8. Gampel A, Mellor H: Small interfering RNAs as a tool to assign Rho GTPase exchange-factor function in vivo. Biochem J 2002, 366:393-398.

9. Karlsson R, Pedersen ED, Wang Z, Brakebusch C: Rho GTPase function in tumorigenesis. Biochim Biophys Acta 2009, 1796:91-98.

10. Liu AX, Rane N, Liu JP, Prendergast GC: RhoB is dispensable for mouse development, but it modifies susceptibility to tumor formation as well as cell adhesion and growth factor signaling in transformed cells. Mol Cell Biol 2001, 21:6906-6912.

11. Adnane J, Muro-Cacho C, Mathews L, Sebti SM, Munoz-Antonia T: Suppression of rho B expression in invasive carcinoma from head and neck cancer patients. Clin Cancer Res 2002, 8:2225-2232.

12. Mazieres J, Antonia T, Daste G, Muro-Cacho C, Berchery D, Tillement V, Pradines A, Sebti S, Favre G: Loss of RhoB expression in human lung cancer progression. Clin Cancer Res 2004, 10:2742-2750.

13. Zhou J, Zhu Y, Zhang G, Liu N, Sun L, Liu M, Qiu M, Luo D, Tang Q, Liao Z, Zheng $Y$, Bi F: A distinct role of RhoB in gastric cancer suppression. Int J Cancer 2011, 128:1057-1068.

14. Lebowitz PF, Davide JP, Prendergast GC: Evidence that farnesyltransferase inhibitors suppress Ras transformation by interfering with Rho activity. Mol Cell Biol 1995, 15:6613-6622.

15. Zalcman G, Closson V, Linares-Cruz G, Lerebours F, Honore N, Tavitian A, Olofsson B: Regulation of Ras-related RhoB protein expression during the cell cycle. Oncogene 1995, 10:1935-1945.

16. Gampel A, Parker PJ, Mellor H: Regulation of epidermal growth factor receptor traffic by the small GTPase rhoB. Curr Biol 1999, 9:955-958.

17. Engel ME, Datta PK, Moses HL: RhoB is stabilized by transforming growth factor beta and antagonizes transcriptional activation. J Biol Chem 1998, 273:9921-9926.

18. Skuli N, Monferran S, Delmas C, Lajoie-Mazenc I, Favre G, Toulas C, CohenJonathan-Moyal E: Activation of RhoB by hypoxia controls hypoxiainducible factor-1a stabilization through glycogen synthase kinase-3 in U87 glioblastoma cells. Cancer Res 2006, 66:482-489.

19. Fritz G, Kaina B, Aktories K: The ras-related small GTP-binding protein RhoB is immediate-early inducible by DNA damaging treatments. J Biol Chem 1995, 270:25172-25177.

20. Canguilhem B, Pradines A, Baudouin C, Boby C, Lajoie-Mazenc I, Charveron M, Favre G: RhoB protects human keratinocytes from UVBinduced apoptosis through epidermal growth factor receptor signaling. J Biol Chem 2005, 280:43257-43263.

21. Srougi $M C$, Burridge $K$ : The nuclear guanine nucleotide exchange factors Ect2 and Net1 regulate RhoB-mediated cell death after DNA damage. PLoS One 2011, 6:e17108.

22. Tang $Y$, Olufemi L, Wang MT, Nie D: Role of Rho GTPases in breast cancer. Front Biosci 2008, 13:759-776.
23. Fritz G, Brachetti C, Bahlmann F, Schmidt M, Kaina B: Rho GTPases in human breast tumours: expression and mutation analyses and correlation with clinical parameters. Br J Cancer 2002, 87:635-644.

24. Jiang WG, Watkins G, Lane J, Cunnick GH, Douglas-Jones A, Mokbel K, Mansel RE: Prognostic value of rho GTPases and rho guanine nucleotide dissociation inhibitors in human breast cancers. Clin Cancer Res 2003, 9:6432-6440.

25. Lane J, Martin TA, Mansel RE, Jiang WG: The expression and prognostic value of the guanine nucleotide exchange factors (GEFs) Trio, Vav1 and TIAM-1 in human breast cancer. Int Semin Surg Oncol 2008, 5:23.

26. Stebel A, Brachetti C, Kunkel M, Schmidt M, Fritz G: Progression of breast tumors is accompanied by a decrease in expression of the Rho guanine exchange factor Tiam1. Oncol Rep 2009, 21:217-222.

27. Ronneburg H, Span PN, Kantelhardt E, Dittmer A, Schunke D, Holzhausen HJ, Sweep FC, Dittmer J: Rho GDP dissociation inhibitor alpha expression correlates with the outcome of CMF treatment in invasive ductal breast cancer. Int J Oncol 36:379-386.

28. Rubino D, Driggers P, Arbit D, Kemp L, Miller B, Coso O, Pagliai K, Gray K, Gutkind S, Segars J: Characterization of Brx, a novel Dbl family member that modulates estrogen receptor action. Oncogene 1998, 16:2513-2526.

29. Su LF, Knoblauch R, Garabedian MJ: Rho GTPases as modulators of the estrogen receptor transcriptional response. J Biol Chem 2001, 276:3231-3237.

30. El Marzouk S, Schultz-Norton JR, Likhite VS, McLeod IX, Yates JR, Nardulli AM: Rho GDP dissociation inhibitor alpha interacts with estrogen receptor alpha and influences estrogen responsiveness. $J$ Mol Endocrinol 2007, 39:249-259.

31. Couderc B, Pradines A, Rafii A, Golzio M, Deviers A, Allal C, Berg D, Penary M, Teissie J, Favre G: In vivo restoration of RhoB expression leads to ovarian tumor regression. Cancer Gene Ther 2008, 15:456-464.

32. Lajoie-Mazenc I, Tovar D, Penary M, Lortal B, Allart S, Favard C, Brihoum M, Pradines A, Favre G: MAP1A light chain-2 interacts with GTP-RhoB to control epidermal growth factor (EGF)-dependent EGF receptor signaling. J Biol Chem 2008, 283:4155-4164.

33. Delfour $C$, Roger $P$, Bret $C$, Berthe ML, Rochaix P, Kalfa N, Raynaud P, Bibeau F, Maudelonde T, Boulle N: RCL2, a new fixative, preserves morphology and nucleic acid integrity in paraffin-embedded breast carcinoma and microdissected breast tumor cells. J Mol Diagn 2006, 8:157-169.

34. Balaguer $P$, Francois $F$, Comunale F, Fenet $H$, Boussioux AM, Pons $M$, Nicolas JC, Casellas C: Reporter cell lines to study the estrogenic effects of xenoestrogens. Sci Total Environ 1999, 233:47-56.

35. Metivier R, Penot G, Hubner MR, Reid G, Brand H, Kos M, Gannon F: Estrogen receptor-alpha directs ordered, cyclical, and combinatorial recruitment of cofactors on a natural target promoter. Cell 2003, 115:751-763.

36. Baron S, Escande A, Alberola G, Bystricky K, Balaguer P, Richard-Foy H: Estrogen receptor alpha and the activating protein-1 complex cooperate during insulin-like growth factor-l-induced transcriptional activation of the pS2/TFF1 gene. J Biol Chem 2007, 282:11732-11741.

37. Petz LN, Ziegler YS, Schultz JR, Kim H, Kemper JK, Nardulli AM: Differential regulation of the human progesterone receptor gene through an estrogen response element half site and Sp1 sites. J Steroid Biochem Mol Biol 2004, 88:113-122.

38. Jiang J, Sarwar N, Peston D, Kulinskaya E, Shousha S, Coombes RC, Ali S: Phosphorylation of estrogen receptor-alpha at Ser167 is indicative of longer disease-free and overall survival in breast cancer patients. Clin Cancer Res 2007, 13:5769-5776.

39. Murphy LC, Niu Y, Snell L, Watson P: Phospho-serine-118 estrogen receptor-alpha expression is associated with better disease outcome in women treated with tamoxifen. Clin Cancer Res 2004, 10:5902-5906.

40. Bulun SE, Lin Z, Zhao H, Lu M, Amin S, Reierstad S, Chen D: Regulation of aromatase expression in breast cancer tissue. Ann N Y Acad Sci 2009, 1155:121-131.

41. Carroll JS, Meyer CA, Song J, Li W, Geistlinger TR, Eeckhoute J, Brodsky AS, Keeton EK, Fertuck KC, Hall GF, Wang Q, Bekiranov S, Sementchenko V, Fox EA, Silver PA, Gingeras TR, Liu XS, Brown M: Genome-wide analysis of estrogen receptor binding sites. Nat Genet 2006, 38:1289-1297. 
42. Wang S, Yan-Neale Y, Fischer D, Zeremski M, Cai R, Zhu J, Asselbergs F, Hampton G, Cohen D: Histone deacetylase 1 represses the small GTPase RhoB expression in human nonsmall lung carcinoma cell line. Oncogene 2003, 22:6204-6213.

doi:10.1186/bcr3377

Cite this article as: Médale-Giamarchi et al.: RhoB modifies estrogen responses in breast cancer cells by influencing expression of the estrogen receptor. Breast Cancer Research 2013 15:R6.

Submit your next manuscript to BioMed Central and take full advantage of:

- Convenient online submission

- Thorough peer review

- No space constraints or color figure charges

- Immediate publication on acceptance

- Inclusion in PubMed, CAS, Scopus and Google Scholar

- Research which is freely available for redistribution

Submit your manuscript at www.biomedcentral.com/submit
C Biomed Central 